\title{
Model order reduction with preservation of passivity, non-expansivity and Markov moments
}

\author{
L. Knockaert ${ }^{1}$, T. Dhaene, F. Ferranti, D. De Zutter \\ Dept. Information Technology, Ghent University \\ Interdisciplinary Institute for Broadband Technology \\ Gaston Crommenlaan 8, PB 201, B-9050 Gent, Belgium.
}

\begin{abstract}
A new model order reduction (MOR) technique is presented which preserves passivity and non-expansivity. It is a projection-based method which exploits the solution of Linear Matrix Inequalities (LMI's) to generate a descriptor state space format which preserves positive-realness and bounded-realness. In the case of both non-singular and singular systems, solving the LMI can be replaced by equivalently solving an algebraic Riccati equation (ARE), which is known to be a more efficient approach. A new ARE and a frequency inversion technique are also presented to specifically deal with the important singular case. The preservation of Markov moments is also guaranteed by the judicious choice of a projection matrix.
\end{abstract}

Key words: Passivity, non-expansivity, positive-real lemma, bounded-real lemma, model order reduction PACS: 84.30.-r, 84.32.-y

2000 MSC: 93B11, 93C05

\section{INTRODUCTION}

The use of model order reduction (MOR) aiming at obtaining compact descriptions of initially large linear state space models has become a standard component in computer-aided design methodologies for a large number of engineering and physics applications. For a good introductory textbook on MOR the reader is referred to [1]. Three MOR approaches can currently be distinguished [2]. The first approach consists of the SVD-based methods, comprising the balanced realization method [3] and Hankel norm approximation [4]. The second approach consists of the projection-based Krylov-subspace methods [5], comprising the Laguerre-SVD approach [6,7]. The third approach consists of iterative methods combining aspects of both the SVD and Krylov methods [8]. In the excellent overview paper [2] both strengths and weaknesses of the three approaches are analyzed; e.g., the first and third approaches generally preserve stability, while the second approach is fast but does not in general guarantee stability (but see also [7]).

Passivity is an important property to satisfy because stable, but non-passive macro-models can produce unstable systems when connected to other stable, even passive, loads. It is well-known that passivity is equivalent with the positive-realness of the system transfer function. The equivalent form of passivity for a scattering matrix representation is non-expansivity or bounded-realness $[9,10]$. It is well established that model reduction techniques with preservation of passivity mostly belong to the balanced truncation class [11-13] or are spectral interpolation-based methods [14-16]. In the case of projection-based Krylov methods the problem of preservation of passivity has been studied by several researchers; for an overview of existing approaches see $[6,17-21]$. The problem with the Krylov-based passivity preserving methods is that they

\footnotetext{
Email addresses: luc.knockaert@intec.ugent.be (L. Knockaert), tom.dhaene@ugent.be (T. Dhaene), francesco.ferranti@intec.ugent.be (F. Ferranti), daniel.dezutter@intec.ugent.be (D. De Zutter)

${ }^{1}$ Corresponding author. This work was supported by a grant of the Research Foundation-Flanders (FWO-Vlaanderen) 
often assume a special descriptor state space setting that may not always be feasible [11].

In this paper, we present a new passivity-preserving and non-expansivity-preserving MOR technique, which does not require any special internal structure of the state space model. It is a projection-based method which exploits the solution of Linear Matrix Inequalities (LMI's) to generate a descriptor state space format which preserves positive-realness and bounded-realness. In the case of both non-singular and singular systems, solving the LMI can be replaced by equivalently solving an algebraic Riccati equation (ARE), which is known to be a more efficient approach [22].

The paper is organized as follows. Section 2 describes the new technique and contains the proof of its passivity-preserving and non-expansivity-preserving properties. Section 3 deals with the important singular case and presents a new ARE and a frequency inversion technique specifically tailored to the singular case. Finally, Section 4 presents pertinent choices for the Krylov projection matrices in such a way that the Markov moments of the system are also preserved.

\section{MAIN RESULTS}

Notation : Throughout the paper $X^{T}$ and $X^{H}$ respectively denote the transpose and Hermitian transpose of a matrix $X$, and $I_{n}$ denotes the identity matrix of dimension $n$. For two Hermitian matrices $X$ and $Y$, the matrix inequalities $X>Y$ or $X \geq Y$ mean that $X-Y$ is respectively positive definite or positive semidefinite. Of course, $X<Y$ or $X \leq Y$ means $Y>X$ or $Y \geq X$. The closed right halfplane $\Re e[s] \geq 0$ is denoted $\mathbb{C}_{+}$.

\subsection{Positive-real systems}

For the real system with minimal realization

$$
\begin{aligned}
& \dot{x}=A x+B u \\
& y=C x+D u
\end{aligned}
$$

where $B \neq 0, C \neq 0$ are respectively $n \times p$ and $p \times n$ real matrices and $A \neq 0$ is a $n \times n$ real matrix, to be passive, it is required that the $p \times p$ transfer function

$$
H(s)=C\left(s I_{n}-A\right)^{-1} B+D
$$

is analytic in $\mathbb{C}_{+}$, such that

$$
H(s)+H(s)^{H} \geq 0 \quad \forall s \in \mathbb{C}_{+}
$$

It is well-known [9] that the positive-real lemma in Linear Matrix Inequality (LMI) format : $\exists P^{T}=P>0$ such that

$$
\left[\begin{array}{cc}
A^{T} P+P A & P B-C^{T} \\
B^{T} P-C & -D-D^{T}
\end{array}\right] \leq 0
$$

guarantees the passivity of the system (1). With the additional stronger condition $D+D^{T}>0$ (strict passivity at $s=\infty$ ), the LMI (2) is feasible if and only if there exists a real matrix $P^{T}=P>0$ satisfying the algebraic Riccati equation (ARE)

$$
A^{T} P+P A+\left(P B-C^{T}\right) W_{p}\left(P B-C^{T}\right)^{T}=0
$$

where

$$
W_{p}=\left(D+D^{T}\right)^{-1}
$$

The ARE (3) is generally solved by constructing the associated Hamiltonian matrix

$$
\mathcal{H}=\left[\begin{array}{cc}
A-B W_{p} C & B W_{p} B^{T} \\
-C^{T} W_{p} C & -A^{T}+C^{T} W_{p} B^{T}
\end{array}\right]
$$


Then the system (1) is passive, i.e., the LMI (2) is feasible, if and only if $\mathcal{H}$ has no purely imaginary eigenvalues [23].

Before tackling the main results, we need to define what is meant by a descriptor state space system. It is a more general system described by the differential equations

$$
\begin{aligned}
E \dot{x} & =A x+B u \\
y & =C x+D u
\end{aligned}
$$

where $E \neq 0$ is a $n \times n$ real matrix called the descriptor. In descriptor state space format the transfer function is given by

$$
H(s)=C(s E-A)^{-1} B+D
$$

Note that it is usually required that $s E-A$ is a regular matrix pencil, i.e., $\operatorname{det}(s E-A)=0$ has a finite number of $s$ values as solutions. In our case we will only need the simple nonsingular descriptor state space format with $E$ nonsingular.

Next suppose $H(s)$ is passive. The following theorem provides a means to obtain a reduced model which preserves passivity.

Theorem 2.1. Suppose the system (1) is passive and let $P=P^{T}>0$ be a solution of the LMI (2). Let $U$ be a $n \times r, 1 \leq r \leq n$ matrix of full rank. Then the reduced descriptor state space system with transfer function

$$
H_{1}(s)=C U\left(s U^{T} P U-U^{T} P A U\right)^{-1} U^{T} P B+D
$$

is passive.

Proof. It is clear that $H_{1}(s)$ can be written as

$$
H_{1}(s)=\tilde{C}\left(s I_{r}-\tilde{A}\right)^{-1} \tilde{B}+D
$$

where

$$
\begin{aligned}
& \tilde{A}=\left(U^{T} P U\right)^{-1} U^{T} P A U \\
& \tilde{C}=C U \quad \tilde{B}=\left(U^{T} P U\right)^{-1} U^{T} P B
\end{aligned}
$$

Putting $\tilde{P}=U^{T} P U$, it is clear that $\tilde{P}^{T}=\tilde{P}>0$. Next consider the matrix

$$
\begin{aligned}
\mathcal{L}_{1} & =\left[\begin{array}{cc}
\tilde{A}^{T} \tilde{P}+\tilde{P} \tilde{A} & \tilde{P} \tilde{B}-\tilde{C}^{T} \\
\tilde{B}^{T} \tilde{P}-\tilde{C} & -D-D^{T}
\end{array}\right] \\
& =\left[\begin{array}{cc}
U^{T}\left(A^{T} P+P A\right) U & U^{T}\left(P B-C^{T}\right) \\
\left(B^{T} P-C\right) U & -D-D^{T}
\end{array}\right]
\end{aligned}
$$

It is easy to show that the matrix $\mathcal{L}_{1}$ can be written as

$$
\mathcal{L}_{1}=\mathcal{E}^{T}\left[\begin{array}{cc}
A^{T} P+P A & P B-C^{T} \\
B^{T} P-C & -D-D^{T}
\end{array}\right] \mathcal{E}
$$

where

$$
\mathcal{E}=\left[\begin{array}{cc}
U & 0_{n \times p} \\
0_{p \times r} & I_{p}
\end{array}\right]
$$

By virtue of the LMI (2) we conclude that $\mathcal{L}_{1} \leq 0$ and the proof is complete. 


\subsection{Bounded-real systems}

For the real system with minimal realization (1) to be non-expansive, it is required that the transfer function $H(s)$ is analytic in $\mathbb{C}_{+}$such that

$$
H(s){ }^{H} H(s) \leq I_{p} \quad \forall s \in \mathbb{C}_{+}
$$

In this case (see [9]), it is well-known that the bounded-real lemma in LMI format : $\exists P^{T}=P>0$ such that

$$
\left[\begin{array}{cc}
A^{T} P+P A+C^{T} C & P B+C^{T} D \\
B^{T} P+D^{T} C & D^{T} D-I_{p}
\end{array}\right] \leq 0
$$

guarantees the non-expansivity of the system (1). With the additional stronger product condition $D^{T} D<I_{p}$ (strict non-expansivity at $s=\infty$ ), the LMI (7) is feasible if and only if there exists a real matrix $P^{T}=P>0$ satisfying the ARE

$$
A^{T} P+P A+C^{T} C+\left(P B+C^{T} D\right) W_{s}\left(P B+C^{T} D\right)^{T}=0
$$

where

$$
W_{s}=\left(I_{p}-D^{T} D\right)^{-1}
$$

The ARE (8) is solved by constructing the associated Hamiltonian matrix

$$
\tilde{\mathcal{H}}=\left[\begin{array}{cc}
A+B W_{s} D^{T} C & B W_{s} B^{T} \\
-C^{T} \tilde{W}_{s} C & -A^{T}-C^{T} D W_{s} B^{T}
\end{array}\right]
$$

where

$$
\tilde{W}_{s}=\left(I_{p}-D D^{T}\right)^{-1}
$$

Then the system (1) is non-expansive, i.e., the LMI (7) is feasible, if and only if $\tilde{\mathcal{H}}$ has no purely imaginary eigenvalues [23].

Suppose $H(s)$ is non-expansive. The following theorem provides a means to obtain a reduced model which preserves non-expansivity.

Theorem 2.2. Suppose the system (1) is non-expansive and let $P=P^{T}>0$ be a solution of the LMI (7). Let $U$ be a $n \times r, 1 \leq r \leq n$ matrix of full rank. Then the reduced descriptor state space system with transfer function

$$
H_{2}(s)=C U\left(s U^{T} P U-U^{T} P A U\right)^{-1} U^{T} P B+D
$$

is non-expansive.

Proof. Similar as Theorem 2.1. It is clear that $H_{2}(s)$ can be written as

$$
H_{2}(s)=\tilde{C}\left(s I_{r}-\tilde{A}\right)^{-1} \tilde{B}+D
$$

where

$$
\begin{aligned}
& \tilde{A}=\left(U^{T} P U\right)^{-1} U^{T} P A U \\
& \tilde{C}=C U \quad \tilde{B}=\left(U^{T} P U\right)^{-1} U^{T} P B
\end{aligned}
$$

Putting $\tilde{P}=U^{T} P U$, it is clear that $\tilde{P}^{T}=\tilde{P}>0$. Next consider the matrix

$$
\begin{aligned}
& \mathcal{L}_{2}=\left[\begin{array}{cc}
\tilde{A}^{T} \tilde{P}+\tilde{P} \tilde{A}+\tilde{C}^{T} \tilde{C} & \tilde{P} \tilde{B}+\tilde{C}^{T} D \\
\tilde{B}^{T} \tilde{P}+D^{T} \tilde{C} & D^{T} D-I_{p}
\end{array}\right] \\
& =\left[\begin{array}{cc}
U^{T}\left(A^{T} P+P A+C^{T} C\right) U & U^{T}\left(P B+C^{T} D\right) \\
\left(B^{T} P+D^{T} C\right) U & D^{T} D-I_{p}
\end{array}\right]
\end{aligned}
$$

It is easy to show that the matrix $\mathcal{L}_{2}$ can be written as

$$
\mathcal{L}_{2}=\mathcal{E}^{T}\left[\begin{array}{cc}
A^{T} P+P A+C^{T} C & P B+C^{T} D \\
B^{T} P+D^{T} C & D^{T} D-I_{p}
\end{array}\right] \mathcal{E}
$$

with $\mathcal{E}$ as in (6). By virtue of the LMI (7) we conclude that $\mathcal{L}_{2} \leq 0$ and the proof is complete. 


\subsection{Markov moment preservation}

In Section 4 we will show how the projection matrix $U$ can be chosen in order to preserve a selection of the so-called Markov moments of the system.

\section{THE SINGULAR CASE}

In the positive-real case the LMI (2) and the ARE (3) are equivalent only in the case $W_{p}>0$ or $D+D^{T}>0$. Similarly, in the bounded-real case the LMI (7) and the ARE (8) are equivalent only in the case $W_{s}>0$ or $D^{T} D<I_{p}$. It is seen that the singular cases $D+D^{T}$ singular or $I_{p}-D^{T} D$ singular cannot easily be solved by means of ARE's (but see also [24] and [25] for that matter), since the pertinent Hamiltonian matrices are then undefined. On the other hand, LMI's are convex formulations and can always be solved by convex optimization [26], without needing ARE solvers and/or Hamiltonian matrices. However, we will show we can say more under sufficiently general conditions and still use the ARE formalism. Our approach differs considerably from the approaches in [24] and [25] in that in our method no state space transformations are needed to obtain the ARE's for the singular case. In order to concentrate solely on the positive-real case we first state the following equivalence Lemmas relating bounded-real and positive-real cases :

Lemma 3.1. $H(s)=C\left(s I_{n}-A\right)^{-1} B+D$ minimal and bounded-real with $A$ Hurwitz is equivalent with $G(s)=C_{1}\left(s I_{n}-A\right)^{-1} B+D_{1}$ positive-real for $C_{1}, D_{1}$ as constructed below.

Proof. We have

$$
I_{p}-H^{T}(-s) H(s) \geq 0 \quad \forall s \in \mathbb{C}_{+}
$$

Now

$$
I_{p}-H^{T}(-s) H(s)=I-D^{T} D-\left(D^{T} C+B^{T} W_{c}\right)\left(s I_{n}-A\right)^{-1} B-B^{T}\left(-s I_{n}-A^{T}\right)^{-1}\left(C^{T} D+W_{c} B\right)
$$

where $W_{c}>0$ is the controllability Grammian.

Hence taking $D_{1}=\left(I-D^{T} D\right) / 2$ and $C_{1}=-D^{T} C-B^{T} W_{c}$ we see that

$$
G^{T}(-s)+G(s) \geq 0 \quad \forall s \in \mathbb{C}_{+}
$$

and the proof is complete.

Lemma 3.2. If $H(s)=C\left(s I_{n}-A\right)^{-1} B+D$ is minimal and bounded-real such that $\operatorname{det}\left[I_{p}-H(s)\right] \neq 0$ for $\Re e[s]>0$ then $G(s)=\left[I_{p}-H(s)\right]^{-1}\left[I_{p}+H(s)\right]=\check{C}\left(s I_{n}-\check{A}\right)^{-1} \check{B}+\check{D}$ is minimal and positive-real with

$$
\begin{aligned}
& \check{A}=A+B\left(I_{p}-D\right)^{-1} C, \quad \check{B}=\sqrt{2} B\left(I_{p}-D\right)^{-1} \\
& \check{C}=\sqrt{2}\left(I_{p}-D\right)^{-1} C, \quad \check{D}=\left(I_{p}-D\right)^{-1}\left(I_{p}+D\right)
\end{aligned}
$$

Conversely, if $G(s)=C\left(s I_{n}-A\right)^{-1} B+D$ is minimal and positive-real then $H(s)=\left[G(s)-I_{p}\right]\left[G(s)+I_{p}\right]^{-1}=$ $\hat{C}\left(s I_{n}-\hat{A}\right)^{-1} \hat{B}+\hat{D}$ is minimal and bounded-real with

$$
\begin{aligned}
& \hat{A}=A-B\left(I_{p}+D\right)^{-1} C, \quad \hat{B}=\sqrt{2} B\left(I_{p}+D\right)^{-1} \\
& \hat{C}=\sqrt{2}\left(I_{p}+D\right)^{-1} C, \quad \hat{D}=\left(D-I_{p}\right)\left(D+I_{p}\right)^{-1}
\end{aligned}
$$

Proof. See [27].

In order to proceed in the singular positive-real case, we first need two more Lemmas : 
Lemma 3.3. If $D+D^{T} \geq 0$ and $\operatorname{rank}\left(D+D^{T}\right)=r<p$ there exists a $p \times p$ orthogonal transformation matrix $\Gamma$ such that

$$
\Gamma^{T}\left(D+D^{T}\right) \Gamma=\left[\begin{array}{cc}
R_{r} & 0 \\
0 & 0
\end{array}\right]
$$

where the $r \times r$ matrix $R_{r}$ is symmetric positive definite. The positive-realness of $\tilde{H}(s)=\Gamma^{T} H(s) \Gamma$ is not affected by this transformation.

Proof. See [24]. Note that $r=0$ corresponds to the totally singular case $D+D^{T}=0$.

Lemma 3.4. Suppose $B$ and $C$ are full rank. Then there exists a matrix $P=P^{T}>0$ that satisfies $P B=C^{T}$ if and only if $C B=B^{T} C^{T}>0$. Furthermore, in that case, all positive definite solutions of $P B=C^{T}$ are given by

$$
P=C^{T}(C B)^{-1} C+B_{\perp} X B_{\perp}^{T}
$$

where $X$ is an arbitrary $(n-p) \times(n-p)$ positive definite matrix and $B_{\perp}$ is the orthonormal null space of $B$.

Proof. See [28]. Note that if $\operatorname{ker}(B)=\{0\}$, which can happen when $p \geq n$, the only solution is $P=$ $C^{T}(C B)^{-1} C$.

The next theorem provides an ARE approach for the singular positive-real case.

Theorem 3.1. Suppose the positive-real singular system is as in Lemma 3.3, i.e.,

$$
D+D^{T}=\left[\begin{array}{cc}
R_{r} & 0 \\
0 & 0
\end{array}\right]
$$

with $R_{r}$ positive definite. Then provided the matrices

$$
C_{s} B_{s}
$$

and

$$
\mathcal{R}=-\left(C_{s} A B_{s}\right)^{T}-C_{s} A B_{s}-\left(C_{s} B_{r}-B_{s}^{T} C_{r}^{T}\right) R_{r}^{-1}\left(B_{r}^{T} C_{s}^{T}-C_{r} B_{s}\right)
$$

(constructively defined in the proof below) are symmetric positive definite, there is a positive definite solution $P$ of the composite algebraic Riccati equation (constructively defined in the proof below) :

$$
A^{T} P+P A+\left(P B_{r}-C_{r}^{T}\right) R_{r}^{-1}\left(P B_{r}-C_{r}^{T}\right)^{T}+\left(P \mathcal{B}-\mathcal{C}^{T}\right) \mathcal{R}^{-1}\left(P \mathcal{B}-\mathcal{C}^{T}\right)^{T}=0
$$

Proof. We start with the LMI formulation by means of the Lur'e equations [29] :

$$
\begin{aligned}
A^{T} P+P A & =-Q^{T} Q \\
P B-C^{T} & =-Q^{T} W \\
D+D^{T} & =W^{T} W
\end{aligned}
$$

Partitioning the matrices $B, C, Q$ and $W$ as

$$
B=\left[B_{r}, B_{s}\right] \quad C=\left[C_{r}^{T}, C_{s}^{T}\right]^{T} \quad Q=\left[Q_{r}^{T}, Q_{s}^{T}\right]^{T} \quad W=\left[\begin{array}{cc}
W_{r} & 0 \\
0 & 0
\end{array}\right]
$$

we can reformulate the Lur'e equations as :

$$
\begin{aligned}
A^{T} P+P A & =-Q_{r}^{T} Q_{r}-Q_{s}^{T} Q_{s} \\
P B_{r}-C_{r}^{T} & =-Q_{r}^{T} W_{r} \\
P B_{s}-C_{s}^{T} & =0 \\
R_{r} & =W_{r}^{T} W_{r}
\end{aligned}
$$


Eliminating equations (11d) and (11b) we obtain

$$
\begin{aligned}
A^{T} P+P A+\left(P B_{r}-C_{r}^{T}\right) R_{r}^{-1}\left(P B_{r}-C_{r}^{T}\right)^{T} & =-Q_{s}^{T} Q_{s} \\
P B_{s}-C_{s}^{T} & =0
\end{aligned}
$$

If the aim were solely to solve equation $(12 \mathrm{~b})$, we could utilize Lemma 3.4, but in general this will not be sufficient (except when $\operatorname{ker}\left(B_{s}\right)=\{0\}$ ), since we also need to satisfy equation (12a). Anyway, a first necessary condition for the existence of a positive definite $P$ is $C_{s} B_{s}=\left(C_{s} B_{s}\right)^{T}>0$ (see also [24]). Next, if we right-multiply equation (12a) with $B_{s}$, we obtain

$$
A^{T} C_{s}^{T}+P A B_{s}+\left(P B_{r}-C_{r}^{T}\right) R_{r}^{-1}\left(B_{r}^{T} C_{s}^{T}-C_{r} B_{s}\right)=-Q_{s}^{T} Q_{s} B_{s}
$$

Defining $W_{s}=Q_{s} B_{s}$ and left-multiplying equation (13) with $B_{s}^{T}$, we obtain

$$
\left(C_{s} A B_{s}\right)^{T}+C_{s} A B_{s}+\left(C_{s} B_{r}-B_{s}^{T} C_{r}^{T}\right) R_{r}^{-1}\left(B_{r}^{T} C_{s}^{T}-C_{r} B_{s}\right)=-W_{s}^{T} W_{s}
$$

Defining

$$
\begin{aligned}
\mathcal{V} & =B_{r}^{T} C_{s}^{T}-C_{r} B_{s} \\
\mathcal{B} & =A B_{s}+B_{r} R_{r}^{-1} \mathcal{V} \\
\mathcal{C} & =-C_{s} A+\mathcal{V}^{T} R_{r}^{-1} C_{r} \\
\mathcal{R} & =-\left(C_{s} A B_{s}\right)^{T}-C_{s} A B_{s}-\mathcal{V}^{T} R_{r}^{-1} \mathcal{V}
\end{aligned}
$$

we can rewrite equations (13) and (14) as

$$
\begin{aligned}
P \mathcal{B}-\mathcal{C}^{T} & =-Q_{s}^{T} W_{s} \\
\mathcal{R} & =W_{s}^{T} W_{s}
\end{aligned}
$$

Assuming $\mathcal{R}$ positive definite, we can write

$$
Q_{s}^{T} Q_{s}=\left(P \mathcal{B}-\mathcal{C}^{T}\right) \mathcal{R}^{-1}\left(P \mathcal{B}-\mathcal{C}^{T}\right)^{T}
$$

yielding the following composite algebraic Riccati equation for $P$ :

$$
A^{T} P+P A+\left(P B_{r}-C_{r}^{T}\right) R_{r}^{-1}\left(P B_{r}-C_{r}^{T}\right)^{T}+\left(P \mathcal{B}-\mathcal{C}^{T}\right) \mathcal{R}^{-1}\left(P \mathcal{B}-\mathcal{C}^{T}\right)^{T}=0
$$

and the proof is complete.

Remark: in the totally singular case $D+D^{T}=0$ the Riccati equation becomes

$$
A^{T} P+P A+\left(P \mathcal{B}-\mathcal{C}^{T}\right) \mathcal{R}^{-1}\left(P \mathcal{B}-\mathcal{C}^{T}\right)^{T}=0
$$

with

$$
\begin{aligned}
\mathcal{B} & =A B \\
\mathcal{C} & =-C A \\
\mathcal{R} & =-(C A B)^{T}-C A B
\end{aligned}
$$

As a last result, which can also help finding the LMI matrix $P$ in the singular case, we have the following :

Theorem 3.2. Frequency inversion theorem : Let $H(s)=C\left(s I_{n}-A\right)^{-1} B+D$ be minimal and positive-real with A Hurwitz. Then $G(s)=\tilde{C}\left(s I_{n}-\tilde{A}\right)^{-1} \tilde{B}+\tilde{D}$ with

$$
\tilde{A}=A^{-1} \quad \tilde{B}=A^{-1} B \quad \tilde{C}=-C A^{-1} \quad \tilde{D}=D-C A^{-1} B
$$

is also positive real and admits the same $P$ matrix as $H(s)$. 
Proof. It is straightforward to see that when $A$ is Hurwitz, then $A^{-1}$ is also Hurwitz and vice versa. Also, it is simple to see by substitution (see also [30]) that $G(s)=H(1 / s)$. By positive-realness, $H(s)$ admits a factorization [29] :

$$
H(s)+H(-s)^{T}=M(-s)^{T} M(s) \quad \forall s \in \mathbb{C}_{+}
$$

Since the mapping $s \mapsto 1 / s$ is one-to-one in (extended) $\mathbb{C}_{+}$, it follows that

$$
G(s)+G(-s)^{T}=H(1 / s)+H(-1 / s)^{T}=M(-1 / s)^{T} M(1 / s) \quad \forall s \in \mathbb{C}_{+}
$$

In other words $G(s)$ is positive-real. To prove it admits the same $P$ as $H(s)$ we write the Lur'e equations

$$
\begin{aligned}
A^{T} P+P A & =-Q^{T} Q \\
P B-C^{T} & =-Q^{T} W \\
D+D^{T} & =W^{T} W
\end{aligned}
$$

Define $\mathcal{Q}=-Q A^{-1}$ and $\mathcal{W}=W-Q A^{-1} B$. It is easy to see that

$$
\tilde{A}^{T} P+P \tilde{A}=-\mathcal{Q}^{T} \mathcal{Q}
$$

Also

and finally

$$
-\mathcal{Q}^{T} \mathcal{W}=A^{-T}\left[Q^{T} W-Q^{T} Q A^{-1} B\right]=P \tilde{B}-\tilde{C}^{T}
$$

$$
\tilde{D}+\tilde{D}^{T}=\mathcal{W}^{T} \mathcal{W}
$$

which completes the proof.

Note that $\tilde{D}=H(0)$ and hence Theorem 3.2 maps the positive-realness problem from $s=\infty$ to $s=0$. Of course it could be that both $H(\infty)+H(\infty)^{T}$ and $H(0)+H(0)^{T}$ are singular, in which case Theorem 3.1 or the approaches in [24] and [25] will provide solutions.

\section{MARKOV MOMENT PRESERVATION}

In the Section 2 we showed that passivity and non-expansivity can be preserved by introducing a full rank matrix $U$. In this section we will show how pertinent column-orthogonal projection matrices $U$ can be constructed which also preserve the so-called Markov moments of the system. To see this, we first write the Laurent expansion of

$$
H(s)=C\left(s I_{n}-A\right)^{-1} B+D=C(s P+G)^{-1} R+D
$$

with $G=-P A, R=P B$, in the vicinity of $s=\infty$.

We have

$$
H(s)=D+\sum_{k=0}^{\infty}(-1)^{k} s^{-k-1} C \Omega^{k} B
$$

where $\Omega=-A$. This can be written as

$$
H(s)=\sum_{k=-1}^{\infty}(-1)^{k} s^{-k-1} \mathcal{M}_{k}
$$

The coefficients $\mathcal{M}_{k}=C \Omega^{k} B, k \geq 0$ and $\mathcal{M}_{-1}=-D$ are known (up to a sign) as the Markov moments of $H(s)$ at $s=\infty$. Next consider the $n \times r$ Krylov matrix $(r=p q \leq n)$

$$
\mathcal{K}=\left[B, \Omega B, \Omega^{2} B, \ldots, \Omega^{q-1} B\right]
$$


and consider choosing an orthonormal basis for the columns of $\mathcal{K}$, which can be implemented by performing the 'thin' SVD of the Krylov matrix as $\mathcal{K}=U \Sigma V^{T}$, and where the $n \times r$ matrix $U$ is column-orthogonal. Putting

$$
\begin{aligned}
& \tilde{P}=U^{T} P U \quad \tilde{G}=U^{T} G U \quad \tilde{R}=U^{T} R \\
& \tilde{C}=C U \quad \tilde{\Omega}=\tilde{P}^{-1} \tilde{G} \quad \tilde{B}=\tilde{P}^{-1} \tilde{R}
\end{aligned}
$$

the new Markov moments are given by

$$
\tilde{\mathcal{M}}_{-1}=\mathcal{M}_{-1}=-D \quad \tilde{\mathcal{M}}_{k}=\tilde{C} \tilde{\Omega}^{k} \tilde{B} \quad k=0,1, \ldots
$$

We are now in a position to prove (see also [31]) :

Theorem 4.1. With the choice of of $U$ as above, the Markov moments are equal up to order $q-1$, i.e., $\tilde{\mathcal{M}}_{k}=\mathcal{M}_{k}$ for $k=0,1, \ldots, q-1$.

Proof. Since we have constructed an orthonormal basis for the columns of $\mathcal{K}$, we can write $\Omega^{k} B=$ $U W_{k}, k=0, \ldots, q-1$, where $W_{k}$ is a $r \times p$ matrix. Note that we have $R=P B=P U W_{0}$ and $\tilde{R}=U^{T} R=U^{T} P U W_{0}=\tilde{P} W_{0}$ and hence $\tilde{B}=\tilde{P}^{-1} \tilde{R}=W_{0}$. Next consider the $n \times n$ matrix

$$
Z=U \tilde{P}^{-1} U^{T} G
$$

By induction, it is easy to prove that $Z^{k} U=U \tilde{\Omega}^{k}$ for $k=0, \ldots, q-1$ and hence

$$
\tilde{\mathcal{M}}_{k}=\tilde{C} \tilde{\Omega}^{k} \tilde{B}=C Z^{k} U W_{0}=C Z^{k} B \quad k=0, \ldots, q-1
$$

There remains to prove that $Z^{k} B=\Omega^{k} B$ for $k=0, \ldots, q-1$. This is clearly the case for $k=0$. Next suppose that $Z^{k} B=\Omega^{k} B$ for some $k$. Then

$$
P^{-1} G Z^{k} B=\Omega^{k+1} B=U W_{k+1}
$$

Pre-multiplying by $U^{T} P$ yields

$$
U^{T} G Z^{k} B=U^{T} P U W_{k+1}=\tilde{P} W_{k+1}
$$

or

$$
W_{k+1}=\tilde{P}^{-1} U^{T} G Z^{k} B
$$

and hence

$$
Z^{k+1} B=U \tilde{P}^{-1} U^{T} G Z^{k} B=U W_{k+1}=\Omega^{k+1} B
$$

which completes the proof.

Recall that by Theorems 2.1 and 2.2, the reduced order model is passive resp. non-expansive, when the original transfer function $H(s)$ is passive resp. non-expansive. Also, one often wishes to have equal Markov moments calculated about another point than infinity, or else to have Markov moments which are coefficients of a Laguerre expansion $[6,7]$. All these possibilities can be dealt with by transforming the Laplace variable $s$ by means of a real Möbius transformation

$$
s=\frac{\alpha u+\beta}{\gamma u+\delta} \quad \alpha \delta-\beta \gamma \neq 0
$$

The resulting transfer function in the $u$-domain is

$$
(\gamma u+\delta) C[u(\alpha P+\gamma G)+(\beta P+\delta G)]^{-1} R+D
$$

Now assuming that $\alpha P+\gamma G$ is nonsingular, we can define the matrices

$$
\hat{B}=(\alpha P+\gamma G)^{-1} R \quad \hat{\Omega}=(\alpha P+\gamma G)^{-1}(\beta P+\delta G)
$$


After construction of a base $\hat{U}$ of the Krylov matrix

$$
\hat{\mathcal{K}}=\left[\hat{B}, \hat{\Omega} \hat{B}, \hat{\Omega}^{2} \hat{B}, \ldots, \hat{\Omega}^{q-1} \hat{B}\right]=\hat{U} \hat{\Sigma} \hat{V}^{T}
$$

the reduced matrices are now

$$
\tilde{P}=\hat{U}^{T} P \hat{U} \quad \tilde{G}=\hat{U}^{T} G \hat{U} \quad \tilde{R}=\hat{U}^{T} R \quad \tilde{C}=C \hat{U}
$$

For example, inserting $\alpha=s_{0}, \beta=\gamma=1, \delta=0$ in (15), we in fact perform a Taylor expansion about $s_{0}$, as in [32], and inserting $\beta=\alpha, \gamma=-1, \delta=1$ in (15), boils down to a scaled Laguerre expansion with scaling factor $\alpha>0$, as in [6, 7]. Of course, by Theorems 2.1 and 2.2, passivity and non-expansivity are always maintained.

\section{CONCLUSION}

We have presented a new model order reduction technique which preserves passivity and non-expansivity. It is a projection-based method which exploits the solution of Linear Matrix Inequalities to generate a descriptor state space format which preserves positive-realness and bounded-realness. In the case of both non-singular and singular systems, solving the LMI can be replaced by equivalently solving an algebraic Riccati equation, which is known to be a faster approach. A new ARE and a frequency inversion technique are presented to specifically deal with the difficult singular case. Last but not least, we have shown how the pertinent column-orthogonal projection matrix can be constructed such that the Markov moments of the system are also preserved.

\section{References}

[1] W. Schilders, Model order reduction: theory, research aspects and applications, Springer, Berlin, 2008.

[2] A. C. Antoulas, D. C. Sorensen, Approximation of large-scale dynamical systems: an overview, Int. J. Appl. Math. Comput. Sci. 11 (5) (2001) 1093-1121.

[3] B. Moore, Principal component analysis in linear systems: Controllability, observability, and model reduction, IEEE. Trans. Autom. Contr. 26 (1) (1981) 17-32.

[4] K. Glover, All optimal Hankel-norm approximation of linear multivariable systems and their $L^{\infty}$-error bounds, Int. J. Control 39 (6) (1984) 1115-1193.

[5] D. L. Boley, Krylov space methods on state-space control models, Circuits Syst. Signal Process. 13 (6) (1994) $733-758$.

[6] L. Knockaert, D. De Zutter, Passive reduced order multiport modeling: the Padé-Laguerre, Krylov-Arnoldi-SVD connection, Int. J. Electron. Commun. (AEÜ) 53 (5) (1999) 254-260.

[7] L. Knockaert, D. De Zutter, Stable Laguerre-SVD reduced order modeling, IEEE Trans. Circuits Syst. I 50 (4) (2003) 576-579.

[8] I. Jaimoukha, E. Kasenally, Implicitly restarted Krylov subspace methods for stable partial realizations, SIAM J. Matrix Anal. Appl. 18 (1) (1997) 633-652.

[9] S. Boyd, L. El Ghaoui, E. Feron, and V. Balakrishnan, Linear Matrix Inequalities in System and Control Theory, SIAM Studies in Applied Mathematics 15, Philadelphia, PA, 1994.

[10] B. Anderson and S. Vongpanitlerd, Network Analysis and Synthesis, NJ: Prentice-Hall, 1973.

[11] J. R. Phillips, L. Daniel, L. M. Silveira, Guaranteed passive balancing transformations for model order reduction, IEEE Trans. Computer-Aided Design 22 (8) (2003) 1027-1041.

[12] S. Gugercin, A. C. Antoulas, A survey of model reduction by balanced truncation and some new results, Internat. J. Control 77 (8) (2004) 748-766.

[13] T. Reis, T. Stykel, Positive real and bounded real balancing for model reduction of descriptor systems, Int. J. Control 83 (1) (2010) 74-88.

[14] D. C. Sorensen, Passivity preserving model reduction via interpolation of spectral zeros, Systems Control Lett. 54 (4) (2005) 347-360.

[15] A. C. Antoulas, A new result on passivity preserving model reduction, Systems Control Lett. 54 (4) (2005) $361-374$.

[16] R. Ionutiu, J. Rommes, A. Antoulas, Passivity preserving model reduction using dominant spectral zero interpolation, IEEE Trans. Computer-Aided Design 27 (12) (2008) 2250-2263.

[17] Z. Bai, R. Freund, A partial Padé-via-Lanczos method for reduced order modeling, Linear Algebra Appl. $332 / 334$ (2001) 139-164.

[18] S.-Y. Kim, N. Gopal, L. Pillage, Time-domain macromodels for VLSI interconnect analysis, IEEE Trans. Computer-Aided Design 13 (1994) 1257-1270. 
[19] J. M. Wang, C.-C. Chu, Q. Yu, E. Kuh, On projection-based algorithms for model-order reduction of interconnects, IEEE Trans. Circuits Systems I 49 (2002) 1564-1585.

[20] A. Odabasioglu, M. Celik, L. T. Pileggi, PRIMA: Passive reduced-order interconnect macromodeling algorithm, IEEE Trans. Computer-Aided Design 17 (8) (1998) 645-654.

[21] P. Feldmann, R. W. Freund, Efficient linear circuit analysis by Padé approximation via the Lanczos process, IEEE Trans. Computer-Aided Design 14 (5) (1995) 639-649.

[22] P. Gahinet, A. Nemirovski, A. J. Laub and M. Chilali, LMI Control Toolbox User's Guide, Version 1, The MathWorks, Inc, 1995.

[23] S. Boyd, V. Balakrishnan, P. Kabamba, A bisection method for computing the $H_{\infty}$ norm of a transfer matrix and related problems, Math. Control Signals Systems 2 (3) (1989) 207-219.

[24] H. Weiss, Q. Wang, J. L. Speyer, System characterization of positive real conditions, IEEE. Trans. Autom. Contr. 39 (3) (1994) $540-544$.

[25] N. Sadegh, J. D. Finney, B. S. Heck, An explicit method for computing the positive real lemma matrices, in: Proc. 33rd Conf. on Decision and Control, 1994, pp. 1464-1469.

[26] S. Boyd and L. Vandenberghe, Convex Optimization, Cambridge Univ. Press, Cambridge, U.K., 2004.

[27] W. M. Haddad, D. S. Bernstein, Robust stabilization with positive real uncertainty: Beyond the small gain theorem, System \& Control Letters 1 (17) (1991) 191-208.

[28] C. H. Huang, P. A. Ioannou, J. Maroulas, M. G. Safonov, Design of strictly positive real systems using constant output feedback, IEEE. Trans. Autom. Contr. 44 (3) (1999) 569-573.

[29] R. F. Curtain, Old and new perspectives on the positive-real lemma in systems and control theory, Z. Angew. Math. Mech. 79 (9) (1999) 579-590.

[30] R. N. Shorten, P. Curran, K. Wulff, E. Zeheb, A note on spectral conditions for positive realness of transfer function matrices, IEEE. Trans. Autom. Contr. 53 (5) (2008) 1258-1261.

[31] L. Knockaert, A note on strict passivity, Systems Control Lett. 54 (9) (2005) 865-869.

[32] R. W. Freund, Krylov-subspace methods for reduced-order modeling in circuit simulation, J. Comput. Appl. Math. 123 (12) (2000) 395-421. 